\title{
Philosophiques
}

\section{Problématique de philosophies morales occidentales}

\section{Simon Laflamme}

Volume 13, numéro 1, printemps 1986

URI : https://id.erudit.org/iderudit/203301ar

DOI : https://doi.org/10.7202/203301ar

Aller au sommaire du numéro

Éditeur(s)

Société de philosophie du Québec

ISSN

0316-2923 (imprimé)

1492-1391 (numérique)

Découvrir la revue

Citer cet article

Laflamme, S. (1986). Problématique de philosophies morales occidentales. Philosophiques, 13(1), 21-38. https://doi.org/10.7202/203301ar

\section{Résumé de l'article}

Les théories éthiques contiennent des contradictions et sont tissées de typologies parce qu'elles sont soumises à la double contrainte de la rationalisation et de la « pertinence ». Parce qu'elles doivent être rationnelles et " pertinentes "; toujours comprises dans des existences individuelles et sociales, variées et variables, elles sont semblables et différentes. d'utilisation que vous pouvez consulter en ligne.

https://apropos.erudit.org/fr/usagers/politique-dutilisation/ 


\title{
PROBLÉMATIQUE DE PHILOSOPHIES MORALES OCCIDENTALES *
}

\author{
par Simon Laflamme
}

\begin{abstract}
RÉSUMÉ. Les théories éthiques contiennent des contradictions et sont tissées de typologies parce qu'elles sont soumises à la double contrainte de la rationalisation et de la «pertinence». Parce qu'elles doivent être rationnelles et « pertinentes »; toujours comprises dans des existences individuelles et sociales, variées et variables, elles sont semblables et différentes.
\end{abstract}

ABSTRACT. Ethical theories include contradictions and are weaved with typologies because they are subjected to the double constraint of rationalization and "relevancy». Because they must be rational and « relevant », always understood in varied and variable, individual and social existences, they are similar and dissimilar.

Qu'ont gagné nos législateurs à choisir cent mille espèces et faits particuliers, et y attacher cent mille lois ? Ce nombre n'a aucune proportion avec l'infinie diversité des actions humaines. La multiplication de nos inventions n'arrivera pas à la variation des exemples. (Montaigne, Essais, III, XIII.)

\section{INTRODUCTION}

Pas de société dans laquelle on ne retrouve à quelque niveau un consensus moral; sans quoi la collectivité se transforme, se désagrège, explose. Une société est formée d'une pluralité d'individus, de groupes, à certains égards différents, à d'autres semblables, entre lesquels des rapports ne peuvent pas ne pas être entretenus directement ou indirectement. A cause de ses déterminations et des caractères de sa composition, à cause de l'inévitabilité de la communication ou de l'échange - dans leurs sens les plus

* Ce titre est celui d'une conférence lue à l'Université d'Ottawa, le 23 mars 1984, dans le cadre de Colloquium. Le texte présenté ici diffère de celui de la conférence par quelques ajouts. 
larges -, à cause des besoins et des motifs du politique, de l'étatique ou de l'idéologique, une société se donne immédiatement des règles par lesquelles elle peut subsister dans la diversité. Dans la mesure où le social est immanent à l'humain, la morale l'est aussi. La morale n'est pas seconde par rapport à la société : l'une et l'autre apparaissent en même temps.

Certes, du fait universel de la morale il n'y a pas à inférer l'existence d'une morale universelle - dont les fondements pourraient être repérés dans une espèce de raison pure, de sens commun ou de droit naturel. Une morale n'est rien sans ce qu'elle exprime et ce langage est foncièrement social, historique. Néanmoins, de Platon à Sartre, il se dégage de différentes philosophies occidentales majeures comme un cadre a priori dont le penseur paraît ne pas pouvoir s'échapper. Ainsi, malgré les variations historiques, malgré les caractéristiques propres à des ensembles sociaux uniques, il semble que la société impose à l'éthique occidentale une matrice à la fois malléable et contraignante. L'ordre du discours éthique se fait propriété sociale et politique.

Or, reconnaissant qu'une étude sur les pensées collectives ne doive perdre de vue ni l'idée de contingence ni celle de nécessité, excluant qu'une solution à un problème sur ce sujet puisse consister en la définition ou bien d'une notion générique, ou bien soit d'une unité, soit d'un phénomène transhistorique, nous nous posons la question suivante: comment peut-on comprendre le fait de la ressemblance des éthiques occidentales et celui de la spécificité de chacune d'elles? Interrogation immense. Elle touche à toute analyse générale sur le rapport entre le discursif, le social et l'historique. Interrogation banale. On sait par avance que l'irréductibilité sera culturelle et historique. Mais nous demandons précisément comment il se fait que le culturel et l'historique sont indispensables à la compréhension d'un fait aussi universel que celui de la morale. Il est fréquemment reconnu que l'universalité de la morale et l'historicité de l'éthique (la morale présentée par le philosophe) soient liées l'une à l'autre. Notre but est de consolider cette position qui, trop souvent, se résume beaucoup plus en une juxtaposition qu'en une corrélation. Pour répondre à notre question (partie 4), nous commençons par mettre en évidence quelques aspects originaux de la littérature morale (partie 2) puis nous construisons un dénominateur commun 
(partie 3) qui servira ultérieurement à trouver en quoi consiste la singularité de chaque éthique.

\section{L'ÉTHIQUE ÉLASTIQUE}

Il n'y a pas à douter que le moraliste soit de bonne foi. Il n'y a pas à ridiculiser le rêve d'une humanité supérieure ${ }^{1}$, d'une société meilleure ${ }^{2}$ qui, dans plusieurs cas, pointe dans l'horizon lointain de son regard - d'autant moins que l'utopie qu'il affiche côtoie souvent des critiques perspicaces (et c'est sûrement là une des raisons pour lesquelles l'éthique se marie si régulièrement avec l'idéologie politique). Mais la littérature morale semble destinée sinon à établir des contradictions, du moins à entretenir la confusion. Ainsi, et ce ne sont là que quelques exemples, Aristote fait du bonheur parfois un état humain, relatif aux conditions matérielles de la vie ${ }^{3}$, parfois une activité d'indépendante perfection ${ }^{4}$ relevant quasiment du divin ${ }^{5}$; pour les stoïciens, Dieu est cause de tout, règle tout, mais l'individu est libre de se conformer ou non à la nature ${ }^{6}$; chez Kant, l'humain est libre mais soumis à

1. Le sage pour Platon, Aristote, le stoïcien; le saint, le croyant pour Saint Augustin, Saint Thomas d'Aquin; le surhomme pour Nietzsche.

2. La république pour Platon; la société naturelle pour les stö̈ciens, stable pour Machiavel, civile pour Hobbes, Locke, Rousseau, rationnelle pour Kant, communiste pour Marx, Sartre.

3. « $[\ldots]$ de toute évidence le bonheur ne saurait se passer des biens extérieurs $[\ldots]$ En effet, il est impossible ou tout au moins difficile de bien faire si l'on est dépourvu de ressources. [... On ne saurait, en effet, être parfaitement heureux si l'on est disgracié par la nature, de naissance obscure, seul dans la vie $[. . . \mid$ Éthnique de Nicomaque, I, VIII, 15-16, traduction, préface et notes par Jean Voilquin, Paris, Garnier-Flammarion, 1965, p. 32-33.

4. «Il en résulte évidemment que le bonheur doit être placé parmi ce qui est souhaitable en soi, et non pour une autre raison, car il n'a besoin de rien pour être complet et il se suffit entièrement à lui-même. » Ibid., X, VI, 2, p. 273.

5. « $[\ldots \mid$ la possibilité de se suffire à soi-même, le loisir, l'absence de fatigue, dans la mesure où elle est réalisable pour l'homme [...] [L'activité de l'esprit] constituera réellement le bonheur parfait, si elle se prolonge pendant toute la durée de sa vie. Car rien ne saurait être imparfait dans les conditions du bonheur. Une telle existence, toutefois, pourrait être au-dessus de la condition humaine. L'homme ne vit plus alors en tant qu'homme mais en tant qu'il possède quelque caractère divin...» Ibid., X, VII, 7-8, p. 277.

6. Plutarque, aussi agaçant quamusant, écrit cette phrase irritée: «Disons-nous donc que les vertus et les vices, les actions droites et les fautes ne dépendent pas de nous ou bien dirons-nous que le destin est en défaut, que son arrêt n'est pas exécuté, que les volontés et les dispositions de Zeus restent sans effet?" «Des contradictions des stoïciens», Les stoïciens, Paris, Gallimard, La Pléiade, 1962, p. 132. 
des lois qui sont sa propre nécessité et à des lois naturelles ${ }^{7}$; Nietzsche jongle avec une volonté de puissance instinctive ${ }^{8}$ et un besoin inné d'obéir ${ }^{9}$; Sartre affirme que l'humain est ontologiquement absolument libre et prétend que l'histoire est soumise à une loi ${ }^{10}$.

On essaie communément de convaincre de l'harmonie dans ces philosophies alléguant quelque composante réconciliante ou interprétant généreusement les propos du moraliste. Cette exégèse fidèle n'arrive cependant pas à résoudre logiquement le paradoxe ; elle parvient à voiler l'innocente antinomie. En réalité, et une Morale de l'ambiguité le consacre, le prouve, l'ambiguité est inhérente à l'éthique; et si, par impossible ? l'équivoque disparaissait, il faudrait s'attendre aux pires exagérations dans les relations politiques. L'éthique n'est pas sophistique. Elle fait ce qu'elle peut et elle doit le faire. Elle serait plutôt sophistiquée.

Le moraliste entend façonner un discours rationnel qui ordonnera les comportements et les pensées d'une population dont la praxis n'est pas que raisonnements. Entre les mours d'une population et sa doctrine morale, il y a inévitablement inadéquation. Celles-là sont toujours plus riches, plus complexes que celle-ci. En quelque sorte l'éthique se tient à mi-chemin entre la philosophie et la praxis, entre la raison analytique et la raison dialectique, dirait Sartre. Le genre moral se veut rigoureux,

7. Un passage parmi d'autres où se débat l'auteur : «Aussi est-ce une tâche à laquelle la philosophie spéculative ne peut se soustraire, que de montrer du moins que ce qui fait que la contradiction qu'elle croit voir est illusoire, c'est que nous concevons l'homme, quand nous le qualifions de libre, en un autre sens et sous un autre rapport que lorsque nous le considérons comme soumis, en tant que fragment de la nature, aux lois de cette nature même; c'est que non seulement les deux choses peuvent fort bien aller ensemble mais encore qu'elles doivent être conçues comme nécessairement unies dans le même sujet... » Fondements de la métaphysique des moeurs, traduction de Victor Delbos, Paris, Delagrave, p. 198. C'est Kant qui souligne.

8. "Car tout instinct aspire à dominer, et en tant que tel cherche à philosopher.» Par-delà le bien et le mal, Paris, Union générale d'éditions, 10/18, 1970, paragraphe 6, p. 28. (C'est Nietzsche qui souligne.) «[...] la vie elle-même est volonté de puissance.»Ibid., paragraphe 13, p. 36.

9. «[...] si, par conséquent, l'obéissance est ce qui a été le mieux et le plus longtemps exercé et cultivé parmi les hommes, on est en droit de présumer que dans la règle chacun de nous possède en lui le besoin inné d'obéir...» Ibid., paragraphe 199, p. 122-123.

10. Contradiction à laquelle l'auteur tente d'apporter une solution par une définition du concept de raison dialectique. 
dense : il nivelle, stabilise, généralise, prédit, simplifie. Or, dans la vie humaine, tout cas, toute situation n'est pas prévisible; or, l'humanité est fuyante, plurielle. C'est à cette réalité mouvante, nombreuse qu'est destinée l'éthique. L'ambiguité, elle, permet au moraliste de réduire l'écart entre sa philosophie et le vécu dans la collectivité. La morale vivante et la philosophie morale s'appellent réciproquement, celle-ci donnant corps à celle-là, celle-là attirant et défiant celle-ci. Mais chacune a ses exigences à elle. Aussi, l'éthique n'est jamais qu'une des régions où la philosophie et la praxis peuvent se croiser et se modifier. Entre la philosophie et la praxis sociale, l'éthique est cette intersection d'une volonté rationalisante et de l'impossibilité pour cette rationalisation de ne pas se faire pertinente. C'est dans cet espace que l'ambivalence peut être subtilement ou ingénument créée ${ }^{11}$, quiètement ou furtivement entendue. Dans ce va-et-vient entre les philosophies des mœurs et les consciences morales - dialectique grâce à laquelle l'éthique peut se faire utilisable - , se référant à sa doctrine, un individu tantôt comprendra qu'il a raison de n'être pas heureux, éprouvé qu'il est par sa maladie ou par le décès d'un amant, tantôt, au contraire, il surmontera de quelque manière sa léthargique tristesse se rendant compte que le bonheur ne dépend pas seulement des « choses extérieures à l'esprit». C'est parce que l'éthique peut répondre à la vie d'une personne que celle-ci peut faire écho à celle-là. Il ne faudrait pas croire, comme pourrait le suggérer cette unique illustration, que l'ambiguïté du discours moral n'a pour raison rien d'autre que la succession ou alternance des sentiments. On doit retenir, comme le montre indiscutablement la psychanalyse, que, dans le psychisme, non seulement tout n'est pas réflexif, mais encore des impressions opposées peuvent agir simultanément.

L'équivoque analytique de l'éthique est sa richesse pratique. Une doctrine des mœurs ne peut pousser tous les acteurs sociaux à se vautrer dans un bonheur suffisant, pas plus qu'elle ne peut

11. Dans «De la vie heureuse», Sénèque écrit ces deux passages: "Qu'un homme véritable ne se laisse ni corrompre ni dominer par les choses extérieures, qu'il n'admire que lui, qu'il ait foi dans son énergie, qu'il soit prêt à l'une ou l'autre fortune..." (Les stö̈ciens, op. cit., p. 730); "C'est d'abord l'arrogance, l'estime exagérée de soi-même, l'enflure qui met au-dessus de tout, l'amour aveugle et inconsidéré de ce qu'on possède (...) Voilà tout ce que bouscule la vertu...» (Ibid., p. 732). 
encourager à la totale jouissance des biens matériels; pour des motifs de pertinence et pour les fins de la société, elle doit autoriser des écarts tout en rappelant à une norme. Tendance à vouloir tout anticiper et impossibilité d'y parvenir, c'est-à-dire impossibilité de parler d'une seule chose; voilà pourquoi l'éthique a besoin des contradictions, voilà pourquoi elle est convaincante : une existence, qui n'est pas logique incarnée, parce qu'elle se laisse guider par une éthique, si diffuse soit-elle, a besoin d'être comprise par cette dernière, de la sentir familière. Telles sont les éthiques dont se dotent les collectivités. Et le moraliste ne peut compenser les ambiguïtés qui s'imposent à lui soit, par la forme de sa présentation ${ }^{12}$, soit par un ton ou un style emphatiques quand il s'exprime sur ce qu'il privilégie ${ }^{13}$, soit par des concepts amphiboles ${ }^{14}$.

L'éthique renferme des confusions. Mais elle commande clairement de faire le bien, non le mal. Parce qu'elle s'adresse à des existences concrètes, elle ne peut disqualifier tout mal intermédiaire ${ }^{15}$ - ce qui risquerait de compromettre son efficacité

12. La plupart des pages des Fondements de la métaphysique des moeurs sont consacrées à la raison, supérieure : le bien est fait par devoir, parce que c'est raisonnable, parce que c'est la loi, parce que la morale est la loi des êtres raisonnables, etc. Evidemment, de telles propositions ne peuvent être soutenues exclusivement, pas plus chez Kant que chez un autre moraliste: «Assurer son propre bonheur est un devoir (au moins indirect); car le fait de ne pas être content de son état, de vivre pressé de nombreux soucis et au milieu de besoins non satisfaits pourrait devenir aisément une grande tentation d'enfreindre ses devoirs» (op. cit., p. 97) ; ailleurs, «Pour qu'un être, qui est à la fois raisonnable et affecté d'une sensibilité, veuille ce que la raison seule prescrit comme devant se faire, il faut sans doute que la raison ait une faculté de lui inspirer un sentiment de plaisir ou de satisfaction, lié à l'accomplissement du devoir... 》 (op. cit., p. 204-205) (dans les deux passages, c'est Kant qui souligne).

13. «[... l'impératif catégorique seul a valeur dune loi pratique, tandis que les autres impératifs ensemble peuvent bien être appelés des principes, mais non des lois de la volonté...» KANT, op. cit., p. 134 ; c'est Kant qui souligne ainsi.

14. Le concept de bonne volonté, chez Kant, rend possible maints jugements qu'on porterait sur une personne dès qu'on voudrait savoir si l'attitude de celle-ci est naturellement ou historiquement déterminée. Il permet, par ailleurs, de maintenir la morale entre le devoir et l'action, c'est-à-dire abstraitement hors de l'empirie, ce qui est nécessaire pour le fondement a priori d'une question qui ne peut pas exclure réellement la pratique.

15. Le Phédon discute de l'antithèse du bien et du mal. Toutefois, cet ouvrage, qui a pour fil conducteur l'âme, se termine par un tableau extraordinaire, sécurisant ou menaçant, où sont peints des fleuves, l'Acheron, le Tartare, et un marais, l'Acherousiade, dans lesquels pourront expier leurs fautes ceux qui ont tenu l' «entre-deux». 
pratique - ; aussi, cherche-t-elle résolument à convaincre de ce que le mal est à l'antipode du bien. Il n'y a pas d'éthique qui ne fasse état, de quelque manière, d'un certain manichéisme. Désireuse de persuader, elle déclare vaguement mais fermement: le bien est sagesse, c'est l'« honnête», l'activité conforme à la vertu ; le mal est déraison, c'est le vice.

Entre la philosophie morale et le vécu moral, il y a décalage ; il n'y a pas rupture: l'éthique ne peut pas ne pas viser à normaliser les comportements. Implicitement, les équivoques de la doctrine ne peuvent pas avoir pour conséquence d'absoudre toute conduite. L'éthique doit se faire souple; elle ne peut pas se faire insignifiante. Elle n'est pas d'autant plus convaincante qu'elle est plus contradictoire. Elle comporte bon nombre de démonstrations serrées - sans lesquelles, par ailleurs, il ne pourrait y avoir d'antinomies - qui, bien que souvent isolées, superficielles, solidifient l'ensemble. L'éthique contient des contradictions parce qu'elle renferme des démonstrations. Ses raisonnements - qui, en s'additionnant, solidifient l'ensemble du discours - mettent en relief certaines questions (l'immortalité de l'âme chez Platon, la volonté de puissance chez Nietzsche) qui émeuvent les acteurs sociaux et déterminent leurs comportements. Son rationalisme érige la conscience des mœurs; celle-ci, devenant plus visible, se désubjectivant, parvient à unifier les individus qui peuvent l'apercevoir. C'est à ces doctrines élevées que se collent les préceptes négatifs de la morale la plus élémentaire: «tu ne tueras point $» .$.

De la somme de ses confusions et de ses argumentations, du total, pourrait-on ajouter, de ses paraboles, de ses analogies, naît de l'éthique un sens général accompagné d'interdictions nettement stipulées.

Pour rendre le discours moral moralisant, le philosophe exploite aussi les typologies. Or, une typologie indique que le réel est à la fois limité et ouvert: le monde se trouve en même temps enfermé par l'analyse et délivré par les parties de celle-ci. Les typologies ont la faculté de cerner tout un secteur de l'être: dans le cas de l'éthique, elles circonscrivent toute une dimension de l'agir humain. Par les termes qu'elles comportent, elles ont aussi la propriété de ne pas emmurer la personne dans le noir. De 
même, la vie humaine ne se laisse pas enclore dans une seule typologie, pour peu que celle-ci se veuille fructueuse. D'où il résulte que les doctrines des mœurs, de façon caractéristique, cherchent à accumuler, à multiplier ces rationalisations qui, le plus souvent, consistent en des dichotomies. Se dresse ainsi une sorte de grillage, quasiment infranchissable qui, dans un vaste territoire, réussit à retenir à peu près toute personne, en toute circonstance, à lui indiquer les limites du permissible. Volontaire/ involontaire, rationnel/instinctuel, vital/appris, naturel/civil, général/particulier, commun/privé, plaisir/bonheur, vice/vertu. Ces typologies aident la philosophie à combler ses lacunes : même si elle n'est pas pourvue d'une vision adéquate de l'humain, elle parvient à l'entourer.

\section{L'ÉTHIQUE RÉDUITE}

Traversée en tous sens par des typologies, l'éthique peut être comprise comme une structure triangulaire dont chacun des sommets agit sur les deux autres et consiste en l'interaction de deux éléments. En effet, d'une part, l'analyse révèle qu'il n'y a pas de doctrine morale dans laquelle on ne repère pas une discussion sur l'universel et le particulier, une promesse soit de récompense, soit de punition, une présentation d'un désirable et d'un inaccessible. Non seulement dans chaque doctrine des énoncés explicites peuvent-ils être observés, mais il appert que les positions occupées par le moraliste sur chacun de ces points ont des répercussions dans l'ensemble du discours, sont la mentalité de celui-ci. D'autre part, une simplification de cette matrice nous apparaît irréalisable si celle-ci doit être à la fois indicative et utile. Chacun des couples d'éléments est requis; chacune de ces copules est si étroitement dépendante des deux autres qu'on ne peut pas la définir rien qu'en elle-même.

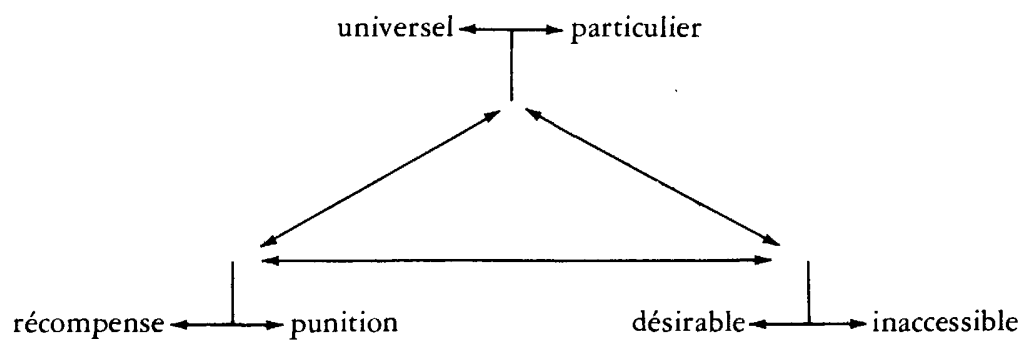




\subsection{L'universel et le particulier}

Rares sont les philosophies qui ne détiennent pas dans la définition même de l'humain, la cause du mal. Rares sont les philosophies qui n'opposent pas raison à passion. Rares sont les philosophies pour lesquelles l'humain n'est pas au moins double, tiraillé, par exemple, entre les tourments corporels et les ravissements de l'intellect ${ }^{16}$.

L'éthique veut que ce qu'elle dit soit valable pour tout humain, du moins pour toute l'humanité à laquelle elle se destine (parfois elle exclut certain collectif). Ceux qu'elle veut convaincre partagent quelque chose: l'âme, la nature humaine, le sens commun, la raison universelle, la loi de l'histoire... Aussi, le moraliste est-il en droit de présumer qu'idéalement les individus agissent et pensent tous identiquement, qu'ils adoptent tous une éthique unique. Souvent, d'ailleurs, c'est sur une notion générique que se fonde la morale. Si les humains ont en commun la raison, l'acte moral est considéré comme rationnel, parce que les mours sont communes. Il faut que les humains soient tous pareils, sans quoi la morale est inconcevable. Il faut par contre que les humains soient tous différents, sans quoi la morale n'est pas nécessaire. Or, dans la mesure où la définition du générique se fait absolue, c'est l'hétérogénéité humaine qui est niée.

Uniformisante, l'éthique ne peut l'être irrémédiablement. Le moraliste distingue entre l'humanité et la personne humaine. D'abord modérément: le particulier est une sous-manifestation du général - et sa mission, son devoir, consiste à disparaître dans l'idéale universalité. Cette hétérogénéisation par le truchement du concept homogénéisant donne lieu à ces jeux qui impliquent une nature humaîne universelle et une nature humaine individuelle, ou encore une raison universelle et une subjectivité. L'individu n'existe qu'en tant qu'il participe (fragment, microsystème) d'une forme suprême. L'éthique parle à tous, mais aussi à chacun. Elle interpelle chacun en tant qu'on est d'une totalité.

16. «Si donc j'étais membre uniquement du monde intelligible, mes actions seraient parfaitement conformes au principe de l'autonomie de la volonté pure; si j'étais seulement une partie du monde sensible, elles devraient être supposées entièrement conformes à la loi naturelle des désirs et des inclinations, par suite à l'hétéronomie de la nature.» KANT, op. cit., p. 193. 
Cependant, l'appartenance au genre n'est pas suffisante pour garantir l'exécution d'un acte conforme à la vertu. Aussi, deuxièmement, le philosophe oppose-t-il la liberté à la non-liberté, au déterminisme. Peu importe la latitude qui est ainsi accordée à l'acteur, le moraliste fait en sorte que la personne ne soit jamais absolument ni responsable ni irresponsable du bien ou du mal qu'elle commet, que, par suite, l'action volontaire soit toujours envisageable, c'est-à-dire que la passivité soit inacceptable. Cet affranchissement du particulier se réalise encore dans une troisième dualité, celle qui sépare le naturel de l'appris. Tout moraliste imagine l'acteur comme étant déterminé à la fois par l'histoire et par la nature. L'essence peut précéder l'existence ou lui succéder. Si la nature n'est pas un résultat historique, un discours circonstanciant minimise les exagérations de celui-ci; si l'éthique part des situations, elle cherche à intégrer les éventualités à une universalisation. Ainsi, le mal ou le bien ont toujours des raisons et extérieures et intérieures à l'individu de sorte que la société n'est jamais entièrement disculpée et que, par conséquent, son contrôle politique peut toujours être perçu comme un moyen et un lieu de la réforme humaine.

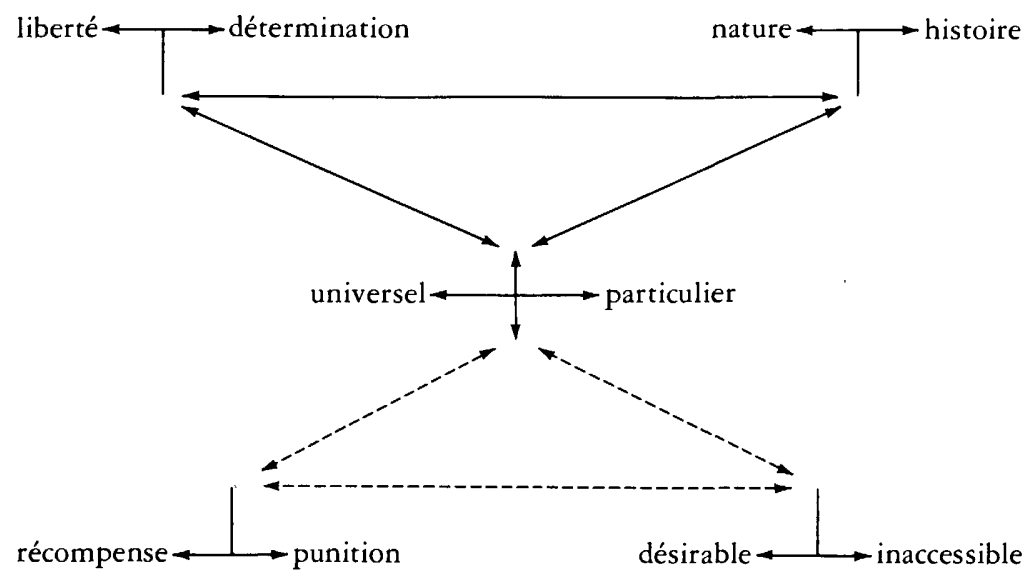

Ce triangle où sont mises en rapport des échelles aux notions interpénétrantes de l'universel et du particulier, de la liberté et de la détermination, du naturel et de l'historique, donne lieu à un foisonnement de possibilités de perceptions et d'interprétations. Dans la majorité des cas, la personne peut constamment se 
rappeler qu'elle est responsable d'elle-même, qu'elle n'est pas irrévocablement coupable. Les adeptes d'une doctrine sont en mesure de porter des jugements sur tout humain, de châtier ou de louer. Le sort de l'humanité peut être amélioré.

La corrélation universel-particulier a des raisons anthropologiques; elle rend le discours uniformisant; elle est possibilité d'interpellation de l'acteur social. Elle est de toute éthique; elle ne rend pas toutes éthiques identiques; elle les rend comparables - et on pourra dire la même chose de tout autre commun dénominateur. Les discours éthiques ne varient pas seulement du point de vue anthropologique qui leur sert de fondement, mais aussi d'après la conception qu'on a du discours à l'intérieur de lui.

\subsection{Récompense et punition}

Le moraliste laisse bien entendre qu'on ne doit pas contourner le mal par crainte de la punition, qu'on ne doit pas pratiquer la vertu dans l'espoir de la récompense. Il faut faire le bien parce que c'est bien. Parce que c'est dans l'ordre des choses; c'est naturel; c'est scientifique. Malgré ces assertions, toute doctrine, qu'elle soit ou non axée sur la récompense et la punition - ce qui ne pourrait être qu'exceptionnel, ce qui ne pourrait rencontrer les exigences de la vie en société - , toute doctrine, donc, signale que quelque bienfait attend le vertueux, que le méchant connaitra quelque malheur. Il y a l'exemple éloquent d'une vie après la mort, de cet enfer, de ce purgatoire ou de ce paradis qui attendent l'âme du défunt. Il y a aussi l'exemple des perspectives plus matérialistes où l'étape ultime de l'histoire est décrite comme un quasi-Eden, où l'avenir humain est celui de la désaliénation. Il n'y a pas d'éthique qui ne promette pas le bonheur : celui auquel s'adresse la doctrine et qui vit en conformité avec son enseignement ou bien connaîtra l'allégresse, ou bien le sort de son existence sera meilleur que celui des autres. Parfois est annoncée une vie comblée par les «plaisirs» terrestres; plus fréquemment est prédit le «vrai bonheur» - à la différence de tout autre. Dans la plupart des philosophies, le bien importe autant que le bonheur (bien et bonheur allant de pair, le bonheur étant immanent à l'acte correct). Or, cette prévision d'enchantement est d'autant plus propice à la persuasion éthique que, pour la majorité des 
moralistes, l'acte vertueux est perçu comme étant plus pénible à accomplir que l'immoral - ce qui, soit dit en passant, n'est pas évident ${ }^{17}$. L'effort doit être récompensé. La noble tâche de l'acteur moral consiste à réprimer le passionnel par le rationnel, à contenir, par la sagesse, de puissantes impulsions, à contrer le corps par l'esprit. On pourrait soupçonner que cette problématique dualiste ne vaille que pour les philosophies dans lesquelles l'épistémologie sépare le sensible de l'intelligible. Poussant cette hypothèse, on pourrait suspecter que la difficulté de la morale sartrienne soit l'effet aussi bien de son approche phénoménologique que de son point de départ situationniste. Cependant, il est à noter que même dans le cas où la doctrine s'assoit sur une épistémologie complexe, l'observateur rencontre tout de même un dualisme grâce auquel un être suprême doit lutter contre un être inférieur : la conscience critique et la conscience aliénée, par exemple. Ce qui est capital pour le moraliste, ce n'est pas tant d'opposer l'esprit au corps que de construire une antithèse d'un heureux bien et d'un misérable mal.

\subsection{Le désirable et l'inaccessible}

Le moraliste encourage à agir avec probité; il dissuade de faire le mal. La personne qui se réfère à son discours ne peut pas prétendre pour elle-même qu'elle est fatalement méchante ou mauvaise; elle ne peut jamais, non plus, imaginer qu'elle est au faîte de la bonté. Le bien se conjuguant avec le bonheur, il n'y a pas, par conséquent, de félicité pour l'être vivant. L'acteur pense qu'il peut toujours mieux faire, que le « réel bonheur» n'est pas atteint dans sa plénitude: il lutte contre la complaisance, il lutte pour lui, pour la gloire de Dieu ou pour celle des humains. L'état idéal selon la doctrine est désirable mais inaccessible. Les personnages merveilleux que le philosophe décrit explicitement ou implicitement et qui représentent, pour l'acteur, des modèles à suivre, sont des figures presque divines, plus qu'humaines. Si bien qu'agisse l'individu, il peut indéfiniment se remémorer les exemples du sage de Sénèque, du communiste de Marx, ces êtres

17. «Une morale de l'effort, écrit Sartre, serait absurde : en quoi l'effort serait-il un signe du bien? Il me coûterait plus d'effort d'étrangler mon fils que de vivre avec lui en bonne intelligence.» Cabiers pour une morale, Paris, Gallimard, 1983, p. 574. 
impossibles à être. Une personne n'est jamais aussi insensible que le Caton de Sénèque mais le portrait de cet homme «invulnérable», qui a existé - qui, donc, par la légende qu'en invente le célèbre précepteur provoque l'illusion d'une réalité. -, chuchote à l'acteur social que les vertus stoïciennes sont des qualités humaines réalisables, qu'on peut être «honnête» dans la cité ; le prolétaire n'éprouve pas dans sa perfection le fusionnement de la conscience et de la théorie du matérialisme historique, mais il fera, imitant autant que cela se peut le modèle du communiste, un virulent critique de la bourgeoisie et un révolutionnaire déterminé. Il faut qu'on veuille être un être qu'on ne pourra jamais être. Le couple désirable-inaccessible et le couple récompense-punition s'appuient l'un sur l'autre. Mais ils n'occupent pas le même espace, ni pratiquement, ni formellement. Ce qui est désirable est récompense quand il est atteint. Mais le désirable renvoie précisément à ce qui ne peut être atteint et l'éthique ne peut abandonner l'acteur dans une complète insatisfaction. La récompense et la punition concernent l'agir; le désirable et l'inaccessible se rapportent à l'être. Il ne suffit pas, pour quelque motif, d'éviter le mal, de faire le bien; il faut encore désirer être bon, sans relâche, car on n'est jamais parfaitement bon.

\section{L'ÉTHIQUE. DES ÉTHIQUES}

L'éthique n'a pas étroitement la tâche d'être logique. Elle doit par-dessus tout être pertinente: elle doit convaincre les acteurs sociaux, à tout moment de leur vie, qu'il faut penser et agir à l'intérieur de certaines limites. Il n'y a pas à s'étonner de ce qu'elle corresponde davantage au discours persuasif qu'au discours démonstratif : parce qu'elle doit être à la fois analyse de l'objet, conscience en l'objet et conscience pour l'objet, parce qu'elle se fait vision du monde, norme collective et volonté intérieure de l'acteur socio-historique, elle ne peut se contenter de la raison théorique.

Toutes les éthiques sont équivalentes dans leur forme. Cependant, toutes ne peuvent pas également persuader une même société.

Rappelons que l'éthique n'est pas la conscience morale. Celle-là, plus réflexive, est toujours simplification. Celle-ci est 
toujours moins réflexive. Ce n'est pas que l'éthique soit obligatoirement seconde par rapport à la conscience morale. Si cela pouvait être utile, nous dirions que le vécu moral est plus essentiel à la société que l'éthique. Mais nous sommes assuré qu'il serait bien simple d'observer qu'ils agissent réciproquement l'un sur l'autre. La philosophie fait la conscience morale; la conscience morale fait la philosophie. Une éthique est l'œuvre d'un philosophe qui pense avec une conscience morale: sa philosophie ne s'impose pas moins à sa conscience morale que celle-ci à celle-là.

La ressemblance entre les éthiques est toute formelle; elle signale néanmoins que le moraliste ne peut pas dire n'importe quoi. Théoriquement, l'éthique n'est que la conséquence, premièrement, de l'impossibilité pour la vie en société de ne pas être morale, dont le corollaire est l'impossibilité pour cette société de ne pas se soumettre à un savoir collectif extérieur aux individus; deuxièmement, de la possibilité de rationaliser ce savoir et de la double contrainte de rationalisation et de pertinence qui en découle. Cette définition tautologique ne signifie pas que les morales soient comparables parce qu'elles sont des expressions particulières de la raison universelle. Il n'y a pas à tirer automatiquement le concept de raison universelle du fait universel de la rationalisation, pas plus qu'on ne dégage nécessairement celui de morale universelle du fait universel de la morale. Et puis, de toute façon, toutes les morales n'ont pas les mêmes raisons. Faut-il alors songer à l'universalité des conditions de la vie en société? En réalité, il faut simplement se rapporter au fait universel de la société. La morale est ce qui fait que la vie en société est possible. Dire: "la société a une morale» ou dire: «la société a une organisation», c'est faire entendre des propositions similaires, des jugements analytiques au sens kantien, c'est-à-dire «dans lesquels l'union du prédicat avec le sujet est pensée par identité » ${ }^{18}$. Il reste à savoir quelles sont cette morale et cette organisation. La structure commune aux discours éthiques, étant donnée la dialectique des mœurs et de la doctrine des mœurs, montre que le citoyen, premièrement, a besoin de comprendre qu'il est unique et membre d'un collectif, deuxièmement, qu'il doit penser qu'il est

18. Critique de la raison pure, traduction de Jules Barni revue par P. Archambault, Paris, Ganier-Flammarion, 1976, p. 63. 
nécessaire, voire avantageux, d'être probe, dangereux de ne pas l'être, troisièmement, que l'illustration d'un modèle à imiter est profitable. Mais elle démontre par-dessus tout que, non insérée dans un contexte, l'éthique est irréelle.

L'éthique concrète est l'éthique intériorisable: celle qui unifie là où l'on doit être unifié; celle qui identifie là où se manifeste une autopersuasion morale. Elle n'est persuasive que dans la mesure où elle est greffée à une histoire, à une société, à une culture, que dans la mesure, donc, où elle peut être conscience réelle. L'éthique, en tant que concrète, s'étend dans l'espace et dans le temps dans la mesure où se déploient ses conditions de possibilité, c'est-à-dire l'aptitude à la comprendre (non pas dans la mesure où se développe le fait de la compréhension!). L'éthique ne peut être intériorisée que parce qu'elle est intégrée à un savoir historique qui la rend compréhensible. Les éthiques sont différentes parce que les sociétés sont variées et variables, aussi bien que les consciences des individus qui les composent, et que ces individus ne peuvent être persuadés que dans des langages audibles, qui correspondent à l'état de leurs consciences constituées. Il n'y aurait de morale universelle que si toutes les consciences étaient constituées pareillement, que si, donc, l'histoire humaine était la même pour tous.

Il ne sert à rien de parler de l'immortalité de l'âme au matérialiste. Le matérialiste a une morale. La notion de l'immortalité de l'âme joue un rôle de premier plan dans la morale platonicienne. Dans l'approche quasi-historique de Hobbes, de Locke, de Rousseau, la notion de passage d'un état de nature à un état de société est primordiale, bien que chaque fois comprise différemment; elle n'a rien à voir avec la morale stö̈cienne. L'idée de nature humaine est indispensable à la morale stoïcienne ; cette nature a priori est évacuée de la perspective sartrienne. Toutes ces éthiques ont des disciples. Certes, la contrainte de pertinence incite à étudier la morale au pluriel. Cela n'implique pas que le commun dénominateur n'ait aucune conséquence en chacune des éthiques. Cette matrice est exigeante. Le moraliste doit produire ou reproduire des idées précises qui se couleront en elle, qu'il injectera dans la culture ou qu'il puisera en celle-ci. Ainsi Platon intègre-t-il à sa doctrine la conception d'une âme immortelle, la renforce-t-il par sa philosophie dans la conscience 
populaire, dans la proportion où sa philosophie s'impose à l'histoire. C'est encore cette structure qui fait qu'un Nietzsche, qui se voudrait le dépassement de Platon - sa tête de Turc -, ne peut pas ne rien avoir en commun avec celui-ci. Bien sûr l'Allemand se détache effectivement du Grec (par l'épistémologie, par l'individualisme, par le subjectivisme, par la morale du plus fort) mais il le rencontre sur d'autres terrains (sublimation de la classe dominante, éloge de la hiérarchie, mépris des basses classes) et ne fait qu'inverser l'ordre des priorités sur d'autres points (dans la dualité passion/raison notamment). Nietzsche découvre la subjectivité dans l'éthique et conclut à l'arbitraire de la morale; Platon aperçoit l'universalité du fait moral et conclut à la raison universelle éthique. Probablement, un Nietzsche, ne serait-ce que par le ton, le genre, bref le langage, ne saurait persuader un platonicien de la Grèce antique. Néanmoins, Platon aussi bien que Nietzsche cadrent dans la structure profonde de l'éthique ${ }^{19}$.

Parce qu'elle veut normaliser les comportements et les pensées d'un collectif, parce qu'elle est immédiatement sociale ${ }^{20}$, l'éthique est forcément politique.

19. i) Platon:

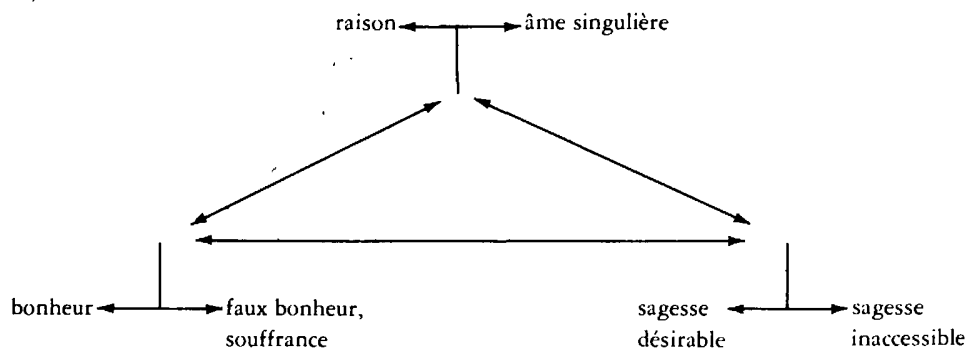

ii) Nietzsche:

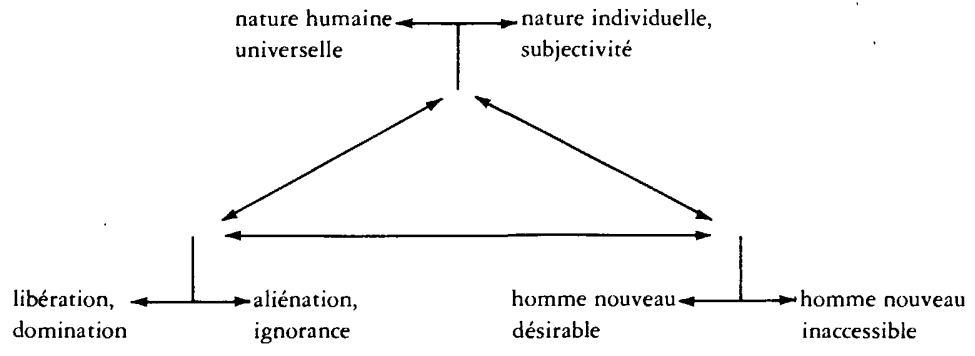

20. Les vertus dominantes dans la littérature morale sont l'honnêteté et la justice. 
Le moraliste sait ce que les humains doivent faire pour que la vie en société soit au mieux. Il connaît l'origine du mal : c'est l'humanité de l'humain: son corps, sa nature, ses passions, ses besoins, ses intérêts, sa mauvaise éducation, son aliénation, la société, la raison, le progrès. Bien qu'il lui soit difficile de ne pas parler d'à peu près tout cela, il a tendance à absolutiser une ou quelques-unes de ces causes - ce qui, par ailleurs, lui permet de canaliser son travail s'il se fait réformateur ou révolutionnaire. C'est parce qu'il sait que l'humanité de l'humain est responsable du mal et qu'il veut extirper le mal de la société que le modèle à imiter qui flotte au-dessus de sa doctrine frôle le divin. Si les humains n'étaient pas ce qu'ils sont, dit-il finalement, il n'y aurait pas de problèmes, ou bien les problèmes ne seraient plus des problèmes. Parce que l'éthique est "persuadeuse», parce qu'elle ne peut pas ne pas être politique, parce qu'il lui est difficile de n'être pas réformatrice, elle croise ou invente fréquemment une idéologie politique (une utopie) ou se glisse en elle (Platon, Cicéron, Machiavel, Hobbes, Locke, Rousseau, Marx, Nietzsche, Sartre). L'éthique n'est pas l'idéologie politique; même si elle n'échappe pas au politique, elle ne se fait pas inéluctablement idéologie politique et c'est pourquoi elle n'a pas toutes les caractéristiques de l'idéologie; mais elle a souvent besoin d'elle (comme de la religion, par ailleurs) pour se déployer parce que l'idéologie fournit à la structure fondamentale des éléments grâce auxquels elle peut exister. Exemple: parce qu'elle cristallise la conscience politique et que, partant, elle unifie un collectif, l'idéologie est pour l'éthique un terrain de prédilection; et puisque l'éthique a elle-même ces fonctions, elle sert, en retour, l'idéologie.

\section{CONCLUSION}

Le moraliste est joueur. Mais son jeu n'a pas de solution idéale. Il n'y a pas de morale ultime. Il y a trop de contraintes et celles-ci ne sont pas maîtrisables. Il faut sans cesse sillonner tous les méandres qui relient la rationalité à la pertinence, le théorique au pratique. Il ne faut pas sortir du labyrinthe; il faut sans cesse le parcourir. Parce que l'éthique se veut persuasive, elle doit rencontrer des consciences historiques. Parce que les histoires, même 
collectives, ne sont pas parfaitement contrôlables, les morales se juxtaposent, se croisent, se superposent, se succèdent.

Une structure universelle de la morale n'a de sens qu'en reconnaissance du fait universel de la rationalisation (et non de la raison) puis du caractère essentiellement «persuadeur» de l'éthique. Dès lors, un fait moral ne peut être étudié que dans une réalité historique, là où se donne une conscience constituée - qui constitue l'histoire. Cependant les conditions de possibilité universelles de l'éthique révèlent que l'incommensurabilité d'une morale n'est pas absolue. A côté de la nécessité de la persuasion, il y a la nécessité de la rationalisation. Il n'y a de raison persuasive morale qu'à l'intérieur des limites de la structure universelle de l'éthique.

C'est en travaillant à des analyses comparatives, toujours en mettant en lumière et l'hétérogénéité et l'homogénéité, qu'on pourra éventuellement découvrir pourquoi Platon, Cicéron, Nietzsche sont compris et incompris par leurs contemporains et par les humains d'époques ultérieures ou de milieux différents, qu'on saisira pourquoi la croyance en l'«évolution de la pensée» n'est pas une explication aux interrogations sur les consciences collectives. Il y a à la source de ces recherches cette question motivante : comment les idées sont-elles communicables et incommunicables d'une société à l'autre, spatialement et temporellement? Nous n'avons pas présenté tout ce qu'il y a d'universel dans la morale. Au sein de la grille qui a été construite, d'autres généralités, sûrement, peuvent être dégagées. Notre tâche a surtout été de contribuer à la délimitation du champ du communicable et de l'incommunicable en éthique. Nous avons pu découvrir qu'une réflexion sur la parenté et l'individualité des éthiques ne peut pas repousser une étude de leur rôle commun de persuader les consciences collectives et, donc, du fait complexe de la compréhension. Notre analyse suggère qu'il n'y a de compréhension morale qu'à l'intérieur d'une structure étroite et précise qui s'ouvre sur une multitude de possibilités.

Département de Sociologie et antbropologie

Université Laurentienne 